\title{
El estrés laboral se asoció a mayor riesgo de mortalidad cardiovascular en trabajadores de la industria
}

Work stress and risk of cardiovascular mortality:prospective cohort study of industrial employees. Kivimäki M, Leino-Arjas $\mathrm{P}$, Luukkonen R. BMJ 2002;325:857.

\begin{abstract}
Objetivo
Examinar la asociación entre el estrés laboral y el riesgo de muerte por enfermedad cardiovascular de acuerdo al modelo de altas demandas laborales y poco control sobre la tarea (en inglés "jobstrain") y de la "disparidad entre el esfuerzo y la recompensa".
\end{abstract}

\section{Diseño}

Estudio de cohorte prospectivo*.

\section{Lugar}

Empleados de una empresa metalúrgica, Finlandia.

\section{Pacientes}

De un total de 4570 fueron seleccionadas 812 personas sin enfermedad cardiovascular, empleadas por al menos 15 meses en 1973-2001. Se obtuvieron los datos a través de cuestionarios, entrevistas y exámenes clínicos.

\section{Evaluación de factores pronósticos}

Los datos de estrés laboral se obtuvieron de auto cuestionarios sobre "job strain" y "balance esfuerzo recompensa", registrándose además variables como sexo, edad, ocupación, tabaquismo, actividad física, presión arterial sistólica (PAS), colesterol total e índice de masa corporal (IMC).

\section{Medición de resultados principales}

Se midió el estrés laboral al inicio del estudio (1973) y se realizó una escala de tres niveles de estrés para ambos cuestionarios (alto, intermedio y bajo) observándose una correlación adecuada entre los dos cuestionarios. Los datos de mortalidad cardiovascular se obtuvieron del registro nacional de mortalidad. El seguimiento promedio fue de 25,6 años con mediciones de colesterol, PAS e IMC a los cinco y diez años.

\section{Resultados principales}

Luego del ajuste por edad y sexo, los empleados con elevado "job strain" tuvieron más del doble de riesgo de muerte cardiovascular que los de bajo "job strain". Lo mismo ocurrió con la valoración del balance entre el esfuerzo y la recompensa (Ver tabla 1). Este riesgo permaneció elevado luego de ajustar por el tipo de trabajo, por las variables biológicas y por los hábitos de vida.

Tabla 1: Asociación entre el grado de estrés laboral y la mortalidad cardiovascular.

\begin{tabular}{|c|c|c|}
\hline \multicolumn{2}{|l|}{ Grado de estrés laboral } & \multirow{2}{*}{$\begin{array}{c}\mathbf{R R}(\mathbf{I C 9 5 \% )}) \\
1 \\
2,2(1,16 \mathrm{a} 4,17)\end{array}$} \\
\hline $\begin{array}{l}\text { Entendido como alta demanda laboral } \\
\text { con poco control sobre la tarea }\end{array}$ & $\begin{array}{l}\text { Bajo } \\
\text { Alto }\end{array}$ & \\
\hline $\begin{array}{l}\text { Entendido como disparidad entre el } \\
\text { esfuerzo y la recompensa* }\end{array}$ & $\begin{array}{l}\text { Bajo } \\
\text { Alto }\end{array}$ & $\begin{array}{c}1 \\
2,36(1,26 \text { a } 4.42)\end{array}$ \\
\hline
\end{tabular}

* Bajo salario, poca aprobación social y bajas oportunidades de progreso.

Analizando los 584 empleados que mantuvieron su tareas sin cambios por cinco años, una alta demanda laboral con poco control sobre la tarea se asoció con casi tres veces mas riesgo (RR 2,9 IC95\% 1,25 a 6,71).Se observó que aquellas personas con valores elevados de estrés aumentaron su colesterol y su IMC, no así su PAS

\section{Conclusiones}

Las altas demandas laborales con poco control sobre la tarea y la disparidad entre el esfuerzo y recompensa parecen ser predictores de la mortalidad cardiovascular.

\section{Comentario}

Este estudio de mortalidad cardiovascular, que pone a prueba la validez de dos de los principales modelos de estrés laboral en una misma población ("job strain" y balance esfuerzo recompensa) mostró que los empleados con alto nivel de estrés tuvieron el doble de riesgo de muerte cardiovascular que sus compañeros con bajo estrés.Dichos hallazgos proporcionan nueva fuerza a la correlación estrés laboral y enfermedad cardiovascular ${ }^{1,2}$.

Lo interesante es que ya en 1973, los autores parecen haber tenido en cuenta las siguientes dificultades:1) no existe un "constructo"* de estrés aceptado por todos los investigadores ${ }^{3,4,5 ; 2)}$ los elementos del medio ambiente interactúan con factores de personalidad a la hora de decidir si un acontecimiento resulta estresante o no para cada individuo ${ }^{3,4,5 ;} 3$ ) para que los efectos del estrés cotidiano se vuelvan evidentes se requiere una exposición sostenida en el tiempo, siendo frecuente que el individuo se adapte a dicha situación, haciendo que lo que inicialmente causaba estrés luego deje de hacerlo ${ }^{3,4,5}$ ("modelos transaccionales del estrés");4) en numerosas ocasiones es necesaria la "sumatoria" de más de un factor generador de estrés ${ }^{3,4,5}$ (modelo de sucesos vitales mayores vs. sucesos menores o "hassles");5) el factor estresante suele provocar una cascada de acontecimientos "intermediarios" (ej. efectos sobre los hábitos alimentarios, adicciones, ejercicio, colestero ${ }^{3,4}$ ). Entre las fortalezas de este trabajo podemos destacar que combina en una misma población dos "constructos" complementarios de estrés laboral ${ }^{1}$ que lo describen desde perspectivas diferentes y que tienen una amplia aceptación entre los investigadores, que pondera efectos "intermediarios" como el impacto en variables biológicas y de comportamiento, que ajusta por potenciales confundidores y, como si fuera poco, cuanta con un seguimiento de 25 años.

Conclusiones del comentador: Si bien aún estamos lejos de demostrar la causalidad, estos hallazgos refuerzan el conocimiento intuitivo de que las condiciones laborales de alto estrés sostenidas en el tiempo se asocian con un aumento de la mortalidad cardiovascular.

\section{Dr. Daniel Sidelski \\ [ Profesor Adjunto a cargo de la titularidad de la materia Corrientes Psicológicas en la Universidad de Morón ] \\ Dr. Guido Bergman [ Staff del Servicio de Prevención Cardiovascular.Instituto Cardiovascular de Buenos Aire ]}

\section{Referencias}

1. Buen Dia J.Estrés laboral y salud, capítulo 6, pag 129-155. Ed.Biblioteca Nueva.Madrid 1998

2. Gonzalez Roma V., Hernández A., Peiro J.y Muñoz P.Estrés de rol e indicadores de riesgo de trastornos cardiovascular.Ansiedad y Estrés 1995, 1 (2-3), 141-156

Implantación de un programa cognitivo-conductual en pacientes hipertensos, Anales de psicología, 1994, 10 (2) 199-216

3.Lazarus R, Folkman S.Estrés y procesos cognitivos Ed Martinez Roca 1986

4. Valdés M.y Flores T. Psicobiología del estrés, conceptos y estrategias de investigación. Ed Martínez Roca 1990

5 Buen Dia, J.Estrés laboral y salud, capítulo 1, pag 21-44. Ed.Biblioteca Nueva.Madrid 1998

Lectura adicional

Peiro J.y Prieto F.Tratado de Psicologìa del Trabajo, cap 7 pag 251-281 Ed Síntesis

Rieg, J Cano Vindel A. Miguel-Tobal J.El estrés laboral bases teóricas y marco de intervención. Ansiedad y Estrés 1995, 1 (2-3) 113-130.

Miguel-Tobal J., Cano Vindel A.Casado Morales M.y Escalona Martinez A.:Emociones e Hipertensión. 